\title{
The impact of lifestyle intervention on left atrial function in type 2 diabetes: results from the DIASTOLIC study
}

\author{
Aseel Alfuhied ${ }^{1,2,3} \cdot$ Gaurav S. Gulsin $^{1,2} \cdot$ Lavanya Athithan $^{1,2} \cdot$ Emer M. Brady $^{1,2} \cdot$ Kelly Parke $^{1,2} \cdot$ Joseph Henson $^{1,2,4}$. \\ Emma Redman ${ }^{1,2,4}$. Anna-Marie Marsh ${ }^{1,2}$. Thomas Yates ${ }^{1,2,4}$. Melanie J. Davies ${ }^{4}$. Gerry P. McCann ${ }^{1,2}$. \\ Anvesha Singh ${ }^{1,2}$ (D)
}

Received: 17 October 2021 / Accepted: 21 February 2022 / Published online: 2 March 2022

(c) The Author(s) 2022

\begin{abstract}
Aerobic exercise training and low energy diets have been shown to improve left ventricular remodelling and diastolic function in adults with type 2 diabetes (T2D), albeit with differential effects. The impact of these lifestyle interventions on left atrial (LA) function, however, has not previously been reported. The DIASTOLIC study was a prospective, randomised, open-label, blind endpoint trial, in which 90 people with obesity and T2D and no prevalent cardiovascular disease were randomised to a 12-week intervention of: (i) routine care, (ii) aerobic exercise training, or (iii) low energy ( $\approx 810 \mathrm{kcal} / \mathrm{day})$ meal replacement plan (MRP). Cardiac magnetic resonance (CMR) imaging was performed pre- and post-intervention. Image analysis included LA volumes (LAV), emptying fraction (LAEF), and LA strain (LAS) corresponding to LA reservoir (LAS-r), conduit (LAS-cd), and booster pump (LAS-bp) function. 73 participants with T2D (mean age $50 \pm 6$ years, $62 \%$ male, body mass index (BMI) $36.1 \pm 5.3 \mathrm{~kg} / \mathrm{m}^{2}$ ) completed the trial and had analysable LA images. There was no significant change in CMR measured LA volumetric function (LAV/LAEF) in any group. The routine care group showed no significant change in BMI or LAS. In the MRP group, there were significant reductions in BMI $\left(4.5 \mathrm{~kg} / \mathrm{m}^{2}\right)$ and a significant increase in LAS-r and LAS-bp (29.9 \pm 7.0 to $32.3 \pm 7.0 \%, p=0.036$ and $14.6 \pm 5.3$ to $17.2 \pm 3.7 \%, p=0.034)$. The exercise group showed a small reduction in BMI $\left(0.49 \mathrm{~kg} / \mathrm{m}^{2}\right)$, with no significant change in LAS. Compared to routine care, weight loss via a 12-week MRP, led to improvements in LA filling and contractile function in adults with T2D and obesity. However, these within-group changes were not statistically significant on between-group comparison.
\end{abstract}

ClinicalTrials.gov Identifier: NCT02590822.

Keywords Left atrium $\cdot$ Type 2 diabetes $\cdot$ Lifestyle $\cdot$ Cardiac magnetic resonance imaging

$\begin{array}{ll}\text { Abbreviations } & \\ \text { LA } & \text { Left atrium } \\ \text { LAEF } & \begin{array}{l}\text { Left atrial emptying fraction } \\ \text { Left atrial strain at booster pump } \\ \text { phase }\end{array} \\ \text { LAS_bp } & \begin{array}{l}\text { Left atrial strain at conduit phase } \\ \text { Left atrial strain at reservoir } \\ \text { phase }\end{array} \\ \text { LAS_cd } & \begin{array}{l}\text { Left atrial volume (maximal/ } \\ \text { minimal/pre-atrial contraction) } \\ \text { LAS_r }\end{array} \\ \text { Type 2 diabetes }\end{array}$

Anvesha Singh

as707@leicester.ac.uk

Extended author information available on the last page of the article

\section{Introduction}

Type 2 diabetes mellitus (T2D) is associated with an increased risk of heart failure (HF) [1] and is the distinct clinical entity of diabetic cardiomyopathy [2]. Diabetic cardiomyopathy is described as myocardial structural or functional abnormality, independent of underlying hypertension, coronary artery disease, or other cardiac diseases [3]. Subclinical left ventricle (LV) diastolic dysfunction is typically the earliest cardiac manifestation of diabetic cardiomyopathy that precedes the occurrence of clinically overt $\operatorname{HF}[2,4,5]$.

LV diastolic dysfunction prolongs relaxation time and diminishes LV passive filling. This leads to reliance on left atrial (LA) contraction at late diastole to achieve optimal LV stroke volume [6]. LA enlargement and dysfunction are associated with LV diastolic dysfunction severity [6-8]. T2D is also associated with LA dysfunction [9], which 
often precede LA dilatation [10], and is independent of LV diastolic dysfunction [11], suggesting its role in reflecting potential evidence of early diabetic cardiomyopathy.

LA strain (LAS) has recently been used to assess LA function, is less load-dependent than volumetric assessment, and could play an important role in classifying LV diastolic dysfunction [12]. LAS has been shown to be an independent predictor of cardiovascular events, superior to LA volumes (LAV) and emptying fraction (LAEF) in the general population [13], patients with HF [14] and chronic kidney disease [15]. It is also a promising non-invasive predictor of elevated LV filling pressure [16, 17]. People with T2D have impaired LA reservoir and conduit function, with increased booster-pump (LA contraction) function by both strain and volumetric assessment, compared to controls [18, 19].

Lifestyle modifications, including improved dietary intake and increased physical activity, are the first-line in and are associated with improved glycaemia, blood pressure $[20,21]$ and reduced the risk of cardiovascular disease [22]. These may also have a role in preventing the onset of clinical HF. Indeed, in a recently completed 12-week randomized controlled trial (DIASTOLIC study), we have shown aerobic exercise improved LV peak early diastolic strain rate (PEDSR), whereas a low-energy meal replacement plan (MRP) improved glycometabolic profiles (achieving remission of T2D in over $80 \%$ ), aortic distensibility, LV concentric remodelling and body weight-corrected peak exercise capacity $\left(\mathrm{VO}_{2}\right)$ [23].

The benefits of these lifestyle interventions on LA function in adults with T2D and obesity are not well established. Our aims for this secondary analysis were: (i) to confirm the impact of T2D and obesity on LA function, and (ii) to investigate the effect of a low-energy MRP and aerobic exercise on LAV and LAS parameters by cardiac magnetic resonance (CMR) imaging, in the DIASTOLIC cohort.

\section{Methodology}

\section{Population}

This is a secondary analysis of the previously published DIASTOLIC study that included T2D participants and age-, sex- and ethnicity-matched controls. This was a prospective, randomised, open-label, blind endpoint trial, to study the effects of lifestyle interventions on cardiovascular structure and function. Participants with T2D and obesity were randomly assigned to receive a 12-week intervention of: (i) routine care, (ii) aerobic exercise training, or (iii) low energy ( $\approx 810 \mathrm{kcal} /$ day) MRP [24]. Key inclusion criteria were: age 18 to 65 years, with established T2D (duration $\geq 3$ months) and body mass index (BMI) $>30 \mathrm{~kg} / \mathrm{m}^{2}$ (or $>27 \mathrm{~kg} / \mathrm{m}^{2}$ if South Asian). Exclusion criteria were presence of significant arrythmia (atrial fibrillation), T2D duration $>12$ years, current treatment with $>3$ glucose-lowering medications or insulin, history/signs/symptoms of cardiovascular disease and weight loss $>5 \mathrm{~kg}$ in the preceding six months. People in the control group were free of T2D, obesity, hypertension and cardiovascular disease. The DIASTOLIC study was ethically approved by the National Research Ethics Service (15/ WM/0222), and all participants provided written informed consent.

Participants with T2D underwent echocardiography and CMR at baseline (prior to randomisation) and 12 weeks. The controls underwent the same investigations at baseline only. The trial protocol and main outcome data have been previously published [23, 24].

\section{CMR}

CMR images were acquired using 1.5T MRI scanner (Siemens Aera, Erlangen, Germany), an 18-channel cardiac coil and retrospective electrocardiographic (ECG) gating, using a standardised protocol, as previously published [24]. This included long (2- and 4-chamber) and short-axis cine images using a steady-state free precession end-expiratory breathhold sequence (typical parameters: voxel size $1.90 \times 1.52$ $\times 8 \mathrm{~mm}$, temporal resolution $48 \mathrm{~ms}$, TR 2.76 and TE 1.15 ) and late gadolinium enhancement (LGE) images at the same slice positions following administration of a total of $0.15 \mathrm{mmol} / \mathrm{kg}$ of gadolinium-based contrast agent (Gadoterate meglumine, Dotarem, Guerbet LLC, France).

\section{CMR image analysis}

Image analysis was performed offline blinded to all participants' details, treatment group and visit type. LV assessment was conducted by G.S.G using cmr42 version 5 (Circle Cardiovascular Imaging, Calgary, Alberta, Canada) as previously described [24], whilst all LA assessment was conducted independently by A.A using Medis v3.1 (Medical imaging systems, Leiden, the Netherlands). Image quality was graded as: $0=$ not analysable, $1=$ fair (artefact present but images still analysable), $2=$ good (artefact present but not in the region of interest), $3=$ excellent.

\section{Left atrial volumetric assessment}

Phasic LA volumes were quantified using the biplane area length method from 2- to 4-chamber cine images [25]. After contouring LA endocardial borders excluding the pulmonary veins and the LA appendage, the LA volume curve throughout the cardiac cycle is automatically generated. The LA volume curve was used to extract LA maximal volume (LAVmax), LA minimal volume (LAVmin) and LA volume pre-atrial contraction (LAVpre-A). The 
maximum and minimum volumes were indexed to body surface area. LA total, passive and emptying fractions were calculated using absolute values of LA volumes as follow:

\section{Statistical analysis}

Statistical tests were performed using SPSS version 26.0 software (Statistical Package for the Social Sciences,

$\mathrm{LA}$ total $\mathrm{EF}($ reservoir function $)=[(\mathrm{LAVmax}-\mathrm{LAVmin}) / \mathrm{LAVmax}] \times 100 \%$,

LA passive $\mathrm{EF}$ (conduitfunction $)=\left[\left(\mathrm{LAVmax}-\mathrm{LAVpre \_ A}\right) / \mathrm{LAVmax}\right] \times 100 \%$,

LA active $\mathrm{EF}$ (booster pump function $)=[($ LAVpre_A-LAVmin $) /$ LAVpre_A $] \times 100 \%$.

\section{Left atrial strain assessment}

LA endocardial borders were traced at ventricular enddiastole and end-systole, excluding the LA appendage and pulmonary veins. The software automatically propagates contours to the rest of the cardiac cycle and formed LA strain curves. Manual correction of contours was performed where required. Using strain curve, LAS at reservoir (LAS_r) and booster-pump (LAS_bp) were extracted, whilst conduit strain (LAS_cd) was calculated as: LAS_ cd =LAS_r - LAS_bp [26]. Global LAS was calculated by averaging segmental strain values from the 2 - and 4-chamber cine images (Fig. 1).
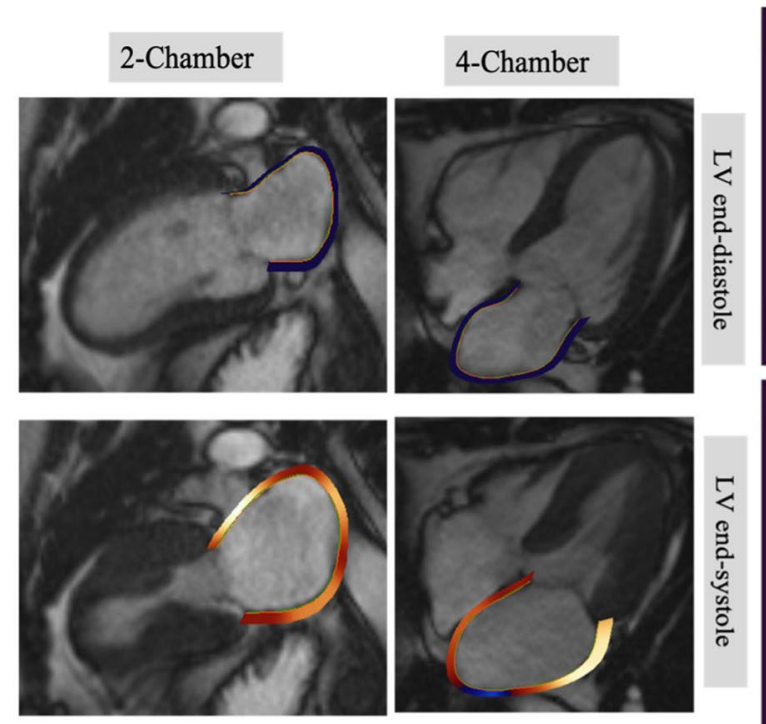

Chicago, IL). Normality was assessed using the Shapiro-Wilk test and histograms. Numerical data are expressed as mean \pm standard deviation (SD). Categorical data are expressed as counts and percentages. At baseline, differences between T2D and controls were evaluated with unpaired t-tests (continuous variables) or Chi-Square test (categorical variables). For continuous variables, One-way analysis of variance (ANOVA) was used to determine significant differences across the three trial groups at baseline.

Data were analysed using generalized linear models to compare the change from baseline to week-12 in the intervention groups relative to the routine clinical care group, adjusted for baseline value (between-group difference). The differences between baseline and week-12 values in each group were also assessed using paired t-test or Wilcoxon

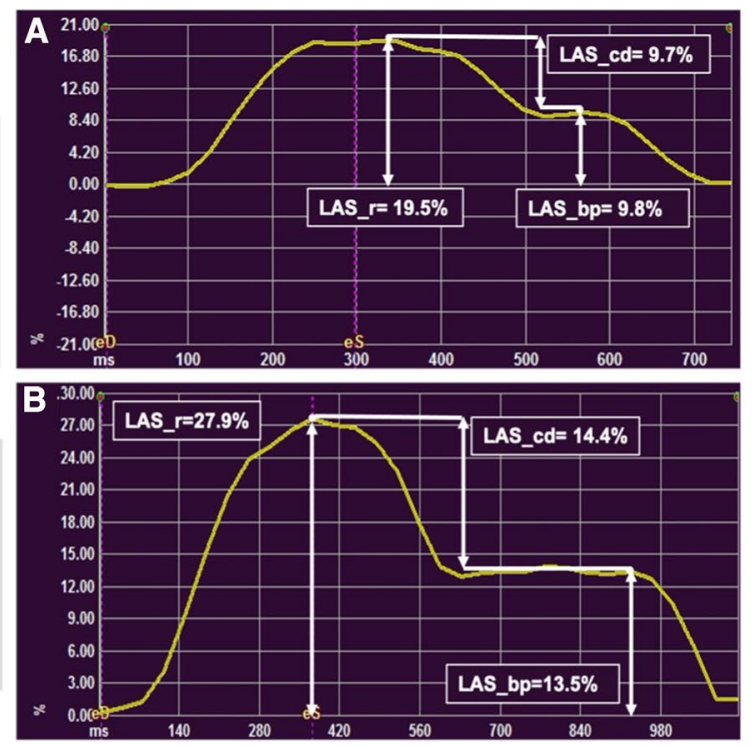

LA strain curve at baseline and B LA strain curve at follow-up. Both curves are for the same patient and produced from tracking LA endocardium across the cardiac cycle using 4-chamber cine. LAS_cd was calculated as: LAS_r - LAS_bp $\left(L A S \_r\right.$ LA strain at reservoir, $L A S_{-}$ $c d$ LA strain at conduit, $L A S_{-} b p$ LA strain at booster-pump phase)
Fig. 1 Left atrial endocardial tracking for strain assessment by feature tracking CMR, with an example of baseline and week-12 left atrial strain for a participant in MRP group. Cine 2- and 4-chamber images illustrating contoured left atrial borders at left ventricular end-diastole (upper) and left ventricular end-systole (lower). On the right, A 
test as appropriate (within-group difference). The latter is included based on the novel nature of the outcomes and in order to support hypothesis generation. It should be interpreted with caution and viewed as secondary to the betweengroup findings. All statistical tests were two-sided, with p-value $<0.05$ was considered statistically significant. Pearson's correlation was used to assess correlation between LA function parameters. Adjustment was not made for multiple comparisons; therefore, data were viewed with caution and in relation to the overall pattern of results.

\section{Results}

Seventy-six participants with T2D completed the trial, and 36 controls were recruited. LA analysis was not possible in 4 scans ( 3 T2Ds and 1 control) due to prospective ECG gating $(n=2)$ and LA foreshortening $(n=2)$. A total of 73 T2D participants (routine care $(n=28)$, exercise $(n=22)$ and $\operatorname{MRP}(n=23))$ and 35 controls were included in the analyses (Fig. 2). All CMR images were analysable $(n=181)$, and image quality was rated as: excellent $(n=148,82 \%)$; good $(n=32,17 \%)$ or fair $(n=1,1 \%)$. At baseline, echocardiographic images for $71(97.3 \%)$ T2D participants had analysable trans-mitral inflow velocities and 67 (91.8\%) had analysable E/e', predominantly due to body habitus.

\section{Baseline characteristics}

Baseline demographics and clinical characteristics of participants and controls are shown in Table 1 . The mean age of participants with T2D was $50 \pm 6$ years and $62 \%$ were men. Healthy controls were well matched for age, sex and ethnicity. Participants with T2D had higher body weight, BMI, blood pressure, heart rate and glycated haemoglobin than controls. Prevalence of hypertension and hyperlipidaemia was higher in participants with T2D. Baseline characteristics, LA and LV parameters in T2D stratified by treatment group are presented in Supplemental Table S1. Overall, there were no significant differences in LA or LV parameters at baseline between the trial groups.

\section{Baseline imaging comparison between T2D and controls}

Baseline LA and LV parameters for participants with T2D versus controls are shown in Table 2 . In comparison to controls, T2D participants had significantly lower LV indexed volumes, higher LVEF and more concentric LV remodelling (higher mass:volume). Echocardiography suggested

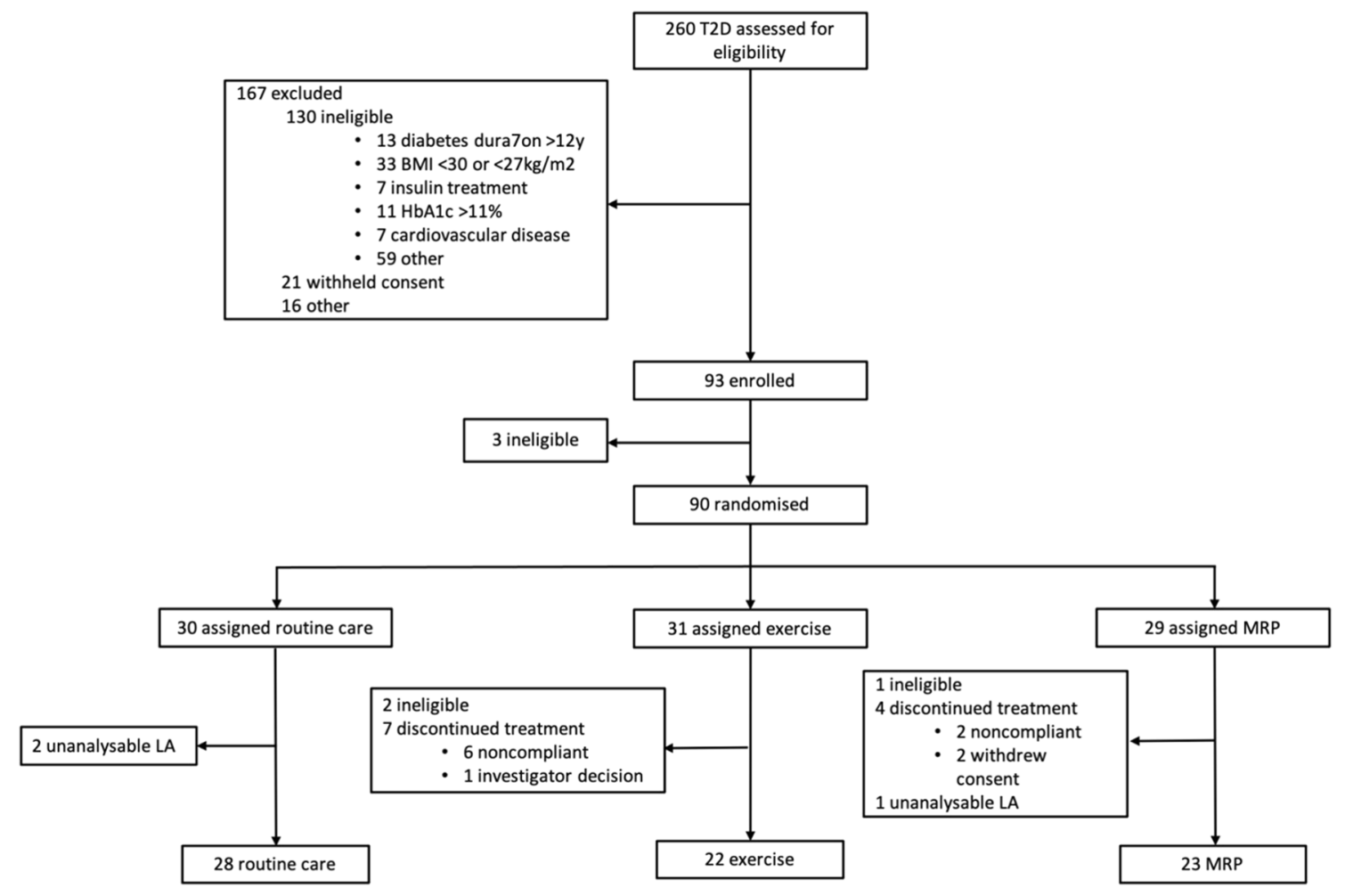

Fig. 2 DIASTOLIC trial profile and number of participants included in the secondary analysis ( $B M I$ body mass index, $L A$ left atrium, $M R P$ meal replacement plan) 
Table 1 Demographics, medical history, and medication of participants with T2D and controls

\begin{tabular}{|c|c|c|c|}
\hline Parameter & $\mathrm{T} 2 \mathrm{D}(\mathrm{n}=73)$ & Controls $(\mathrm{n}=35)$ & $p$-value \\
\hline Age, years & $50.4 \pm 6.3$ & $48.6 \pm 6.3$ & 0.170 \\
\hline Sex, $\mathrm{n}(\%)$ males & $45(61.6 \%)$ & $19(56.3 \%)$ & 0.727 \\
\hline Height, $\mathrm{cm}$ & $168.8 \pm 9.4$ & $169.3 \pm 9.4$ & 0.778 \\
\hline Weight, $\mathrm{kg}$ & $102.6 \pm 15.9$ & $70.4 \pm 10.9$ & $<0.001^{*}$ \\
\hline BMI, $\mathrm{kg} / \mathrm{m}^{2}$ & $36.1 \pm 5.3$ & $24.5 \pm 2.4$ & $<0.001^{*}$ \\
\hline $\mathrm{SBP}, \mathrm{mmHg}$ & $139.8 \pm 15.1$ & $121.2 \pm 13.4$ & $<0.001^{*}$ \\
\hline DBP, $\mathrm{mmHg}$ & $87.8 \pm 7.6$ & $76.5 \pm 7.3$ & $<0.001^{*}$ \\
\hline $\mathrm{HR}$, beats/min & $74.4 \pm 9.8$ & $61.8 \pm 9.9$ & $<0.001^{*}$ \\
\hline \multicolumn{4}{|l|}{ Medical history } \\
\hline Diabetes duration, months & $65.6 \pm 39.1$ & N/A & N/A \\
\hline Hypertension, n (\%) & $36(49.3 \%)$ & $0(0)$ & $<0.001^{*}$ \\
\hline Hyperlipidaemia, n (\%) & $44(60.3 \%)$ & $0(0)$ & $<0.001^{*}$ \\
\hline \multicolumn{4}{|l|}{ Fasting blood tests } \\
\hline Glucose, $\mathrm{mmol} / \mathrm{L}$ & $8.4 \pm 2.47$ & $5.1 \pm 0.48$ & $<0.001^{*}$ \\
\hline $\mathrm{HbA} 1 \mathrm{c}, \%$ & $7.3 \pm 1.03$ & $5.4 \pm 0.24$ & $<0.001^{*}$ \\
\hline \multicolumn{4}{|l|}{ Medications } \\
\hline ACE inhibitor, $\mathrm{n}(\%)$ & $21(28.8 \%)$ & $0(0)$ & $<0.001^{*}$ \\
\hline $\mathrm{ARB}, \mathrm{n}(\%)$ & $10(13.7 \%)$ & $0(0)$ & $0.022 *$ \\
\hline Beta blocker, n (\%) & $4(5.5 \%)$ & $0(0)$ & 0.158 \\
\hline Calcium channel blocker, n (\%) & $16(21.9 \%)$ & $0(0)$ & $0.003 *$ \\
\hline Statin, n (\%) & $47(64.4 \%)$ & $0(0)$ & $<0.001 *$ \\
\hline Metformin, n (\%) & $71(97.3 \%)$ & N/A & N/A \\
\hline Sulfonylurea, n (\%) & $11(15.1 \%)$ & N/A & N/A \\
\hline DPP-IV inhibitor, n (\%) & $14(19.2 \%)$ & N/A & N/A \\
\hline SGLT2 inhibitor, $\mathrm{n}(\%)$ & $9(12.3 \%)$ & N/A & N/A \\
\hline GLP-1 receptor agonist, $\mathrm{n}(\%)$ & $8(11.0 \%)$ & N/A & N/A \\
\hline
\end{tabular}

Data represented as mean $\pm \mathrm{SD}$ or number $(\%)$

$S B P$ systolic blood pressure, $D B P$ diastolic blood pressure, $H R$ heart rate, $A C E i$ angiotensin converting enzyme inhibitor, $A R B$ angiotensin-receptor blocker, $C C B$ calcium channel blocker, DPP-IV dipeptidyl peptidase-IV, SGLT2 sodium glucose cotransporter-2, GLP-1 glucagon-like peptide-1

*Indicates a significant difference with $\mathrm{p}<0.05$ diastolic dysfunction (lower E/A) and higher LV filling pressures $\left(\mathrm{E} / \mathrm{e}^{\prime}\right)$ in T2D. LA indexed volumes and passive $\mathrm{EF}$ were lower in T2D than controls. However, active EF was higher in people with T2D, resulting in no difference in total LAEF between the two groups. Both reservoir and conduit LAS were lower in people with T2D $(31.4 \pm 7.4$ vs $39.8 \pm 10.8 \%, \mathrm{p}<0.001$ and $15.9 \pm 5.5$ vs $24.1 \pm 9.5 \%$, $\mathrm{p}<0.001$, respectively). There was no difference in booster pump LAS between groups.

\section{Change in anthropometrics and LV parameters post-lifestyle intervention}

Changes in anthropometric and LV parameters from baseline to week-12 are shown in Supplemental Table S2. The MRP group demonstrated a significant reduction in weight, $\mathrm{BMI}$, fasting glucose, $\mathrm{HbA} 1 \mathrm{c}$ and systolic blood pressure (SBP), whilst there were no significant changes noted in the exercise group. SBP was also reduced in the standard care group, driven by up-titration of guideline-based antihypertensive medications. There were no significant changes in the LV volumes, EF or mass in the standard care or exercise groups. The MRP group showed a significant increase in LV end-diastolic volume index (LVEDVi), with a corresponding decrease in LVEF, though remaining within normal range.

\section{Change in LA parameters post-lifestyle intervention}

The LA volumetric and strain parameters by CMR at baseline and 12 weeks are shown in Table 3. On betweengroup analysis, corrected for baseline values, there were no statistically significant changes for any LA parameter, relative to the standard care group $(p>0.117)$. However, within-group analysis showed a significant increase in the maximal LAVi of borderline significance in the MRP group $\left(36.2 \pm 9.2\right.$ to $\left.40.2 \pm 13.4 \mathrm{~mL} / \mathrm{m}^{2}, \mathrm{p}=0.06\right)$. The MRP 
Table 2 Baseline LA and LV assessment parameters of participants with $\mathrm{T} 2 \mathrm{D}$ versus controls

\begin{tabular}{|c|c|c|c|}
\hline Parameter & $\mathrm{T} 2 \mathrm{D}(\mathrm{n}=73)$ & Controls $(\mathrm{n}=35)$ & $p$-value \\
\hline \multicolumn{4}{|c|}{ Cardiac magnetic resonance imaging } \\
\hline \multicolumn{4}{|l|}{ Volumetric assessment } \\
\hline $\mathrm{LAVi}_{\mathrm{Max}}, \mathrm{ml} / \mathrm{m}^{2}$ & $33.7 \pm 8.0$ & $43.3 \pm 10.8$ & $<0.001^{*}$ \\
\hline $\mathrm{LAVi}_{\mathrm{Min}}, \mathrm{ml} / \mathrm{m}^{2}$ & $14.9 \pm 5.2$ & $18.6 \pm 5.7$ & $0.002 *$ \\
\hline LA total EF, $\%$ & $56.4 \pm 7.6$ & $57.3 \pm 5.0$ & 0.508 \\
\hline LA passive EF, $\%$ & $27.4 \pm 8.5$ & $34.5 \pm 7.8$ & $<0.001 *$ \\
\hline LA active EF, \% & $39.9 \pm 7.7$ & $34.6 \pm 6.6$ & $0.001 *$ \\
\hline LV EDVi, $\mathrm{ml} / \mathrm{m}^{2}$ & $67.7 \pm 10.2$ & $83.2 \pm 18.9$ & $<0.001^{*}$ \\
\hline LV ESVi, ml/m² & $21.9 \pm 6.4$ & $29.5 \pm 9.1$ & $<0.001^{*}$ \\
\hline LV EF, \% & $68.0 \pm 6.8$ & $65.0 \pm 4.9$ & $0.012^{*}$ \\
\hline LV mass, $\mathrm{g}$ & $123.0 \pm 24.6$ & $107.0 \pm 32.8$ & $0.014^{*}$ \\
\hline $\mathrm{LV}$ mass index, $\mathrm{g} / \mathrm{m}^{2}$ & $55.9 \pm 8.7$ & $58.1 \pm 13.8$ & 0.381 \\
\hline $\begin{array}{l}\text { LV mass/volume, } \\
\mathrm{g} / \mathrm{ml}\end{array}$ & $0.83 \pm 0.11$ & $0.70 \pm 0.10$ & $<0.001 *$ \\
\hline \multicolumn{4}{|l|}{ LA strain } \\
\hline LAS_r, \% & $31.4 \pm 7.4$ & $39.8 \pm 10.8$ & $<0.001^{*}$ \\
\hline LAS_cd, \% & $15.9 \pm 5.5$ & $24.1 \pm 9.5$ & $<0.001^{*}$ \\
\hline LAS_bp, $\%$ & $15.5 \pm 4.9$ & $15.6 \pm 5.4$ & 0.867 \\
\hline \multicolumn{4}{|l|}{ Echocardiography } \\
\hline E-wave, $\mathrm{m} / \mathrm{s}$ & $0.67 \pm 0.13$ & $0.66 \pm 0.13$ & 0.820 \\
\hline A-wave, $\mathrm{m} / \mathrm{s}$ & $0.71 \pm 0.15$ & $0.56 \pm 0.11$ & $<0.001^{*}$ \\
\hline E/A ratio & $0.96 \pm 0.19$ & $1.21 \pm 0.25$ & $<0.001 *$ \\
\hline Average E/e' ratio & $8.69 \pm 2.5$ & $6.40 \pm 1.6$ & $<0.001 *$ \\
\hline
\end{tabular}

Data represented as mean $\pm \mathrm{SD}$

$L A V i_{\text {max }}$ left atrial maximum volume index, $L A V i_{\text {min }}$ left atrial minimum volume index, $L A E F$ left atrial emptying fraction, $L V E D V i$ left ventricular end-diastolic volume index, $L V E S V i$ left ventricular endsystolic volume index, $L V E F$ left ventricular ejection fraction, $L A S \_r$ left atrial strain at reservoir phase, $L A S \_c d$ Left atrial strain at conduit phase, $L A S \_b p$ left atrial strain at booster pump phase

*Indicates a significant difference with $\mathrm{p}<0.05$

group also demonstrated a statistically significant increase in LAS at both reservoir and booster pump phases $(29.9 \pm 7.0$ to $32.3 \pm 7.0 \%, \mathrm{p}=0.036$ and $14.6 \pm 5.3$ to $17.2 \pm 3.7 \%$, $\mathrm{p}=0.034$, respectively) (Fig. 3). There was no change in any strain parameter in the standard care group, and a trend towards an increase in the booster-pump LAS in the exercise group $(15.3 \pm 4.2$ to $17.1 \pm 5.4 \%, \mathrm{p}=0.09)$.

\section{Correlation}

Our previous publication showed significant correlations between volumetric and strain parameters corresponding to LA phasic function [26]. In this study, we investigated the association between the diastology parameters by echocardiography and LA function parameters by CMR. There were significant correlations of average-e' with conduit LAS and passive LAEF, and A-wave with booster-pump LAS (Supplemental Fig. S1).

\section{Discussion}

To our knowledge, this is the first study to investigate the impact of lifestyle interventions on LA strain parameters in adults with T2D and obesity. On within-group analysis, a low-energy MRP led to significant reductions in BMI, $\mathrm{BP}$ and hyperglycaemia, with a corresponding significant increase in reservoir and booster-pump LAS, despite no significant change in conventional LA volumetric parameters or echocardiographic measures of diastolic function. However, these changes were no longer significant when between-group interaction was taken into account.

\section{LA volumes and function in T2D}

Previous LAV data in people with T2D are conflicting, with some studies showing larger LA volumes compared to controls [11, 27], whilst others show it to be smaller [28, 29]. These findings may reflect duration of disease as well as the effect of indexing volumes. In our study, participants with T2D had lower LAVi than controls, in line with previous studies comparing adults with and without T2D from UK Biobank [28] and in heart failure with preserved ejection fraction (HFpEF) patients with T2D [30, 31].

LA function is recognized as a predictor of HF hospitalization and adverse outcomes across a range of cardiovascular diseases [32-36]. Our results show people with T2D had lower passive LAEF corresponding to LA conduit function, which may reflect reduced LV compliance [10, 37]. This was in conjunction with impaired LV relaxation as mitral E/A was also lower. Consequently, active LAEF was higher in people with T2D to compensate for the reduction in passive LAEF, as shown previously [37]. This phenomenon has also been seen in early stages of hypertensive heart disease [38], however, absent in cases where the LV filling pressure is chronically elevated such as in HFpEF [39]. Accordingly, we observed that our asymptomatic participants with $\mathrm{T} 2 \mathrm{D}$ were at early stages of LV diastolic dysfunction with grade-1 LV diastolic dysfunction.

People with T2D also showed impaired conduit LAS. In addition, LA filling was reduced as measured by a reduction in reservoir LAS. This finding may support previous studies where LAS detects subclinical reservoir dysfunction in people with T2D, even in those with normal LA volume, suggesting an early impairment in LA reservoir function [10, 40]. A previous study also demonstrated lower reservoir and conduit LAS in younger adults with obesity compared with normal-weight volunteers [41]. 


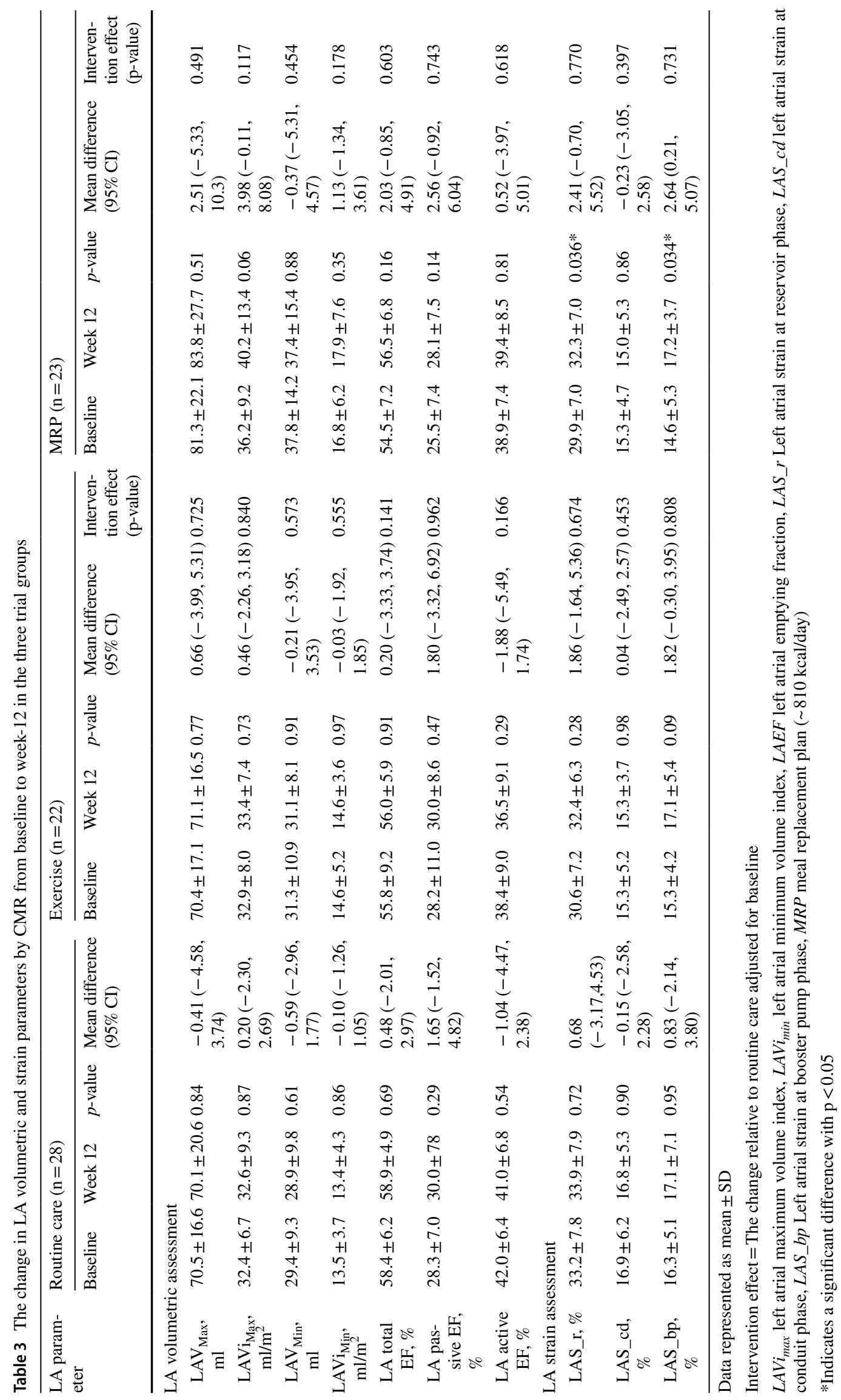


Fig. 3 Changes in BMI, reservoir and booster-pump LAS in participants with T2D after 12 weeks of lifestyle intervention. Line graph representing change of body mass index (BMI) (blue), left atrial strain at reservoir (LAS_r) (Red), and left atrial strain at booster-pump (LAS_bp) (Green). Changes post lifestyle intervention (x-axis) at baseline and week-12 post routine care (A), exercise (B) and meal replacement plan (MRP) (C). Means are shown with error bars depicting standard error of the mean (y-axis) (*Indicates a significant difference with $\mathrm{p}<0.05$ )

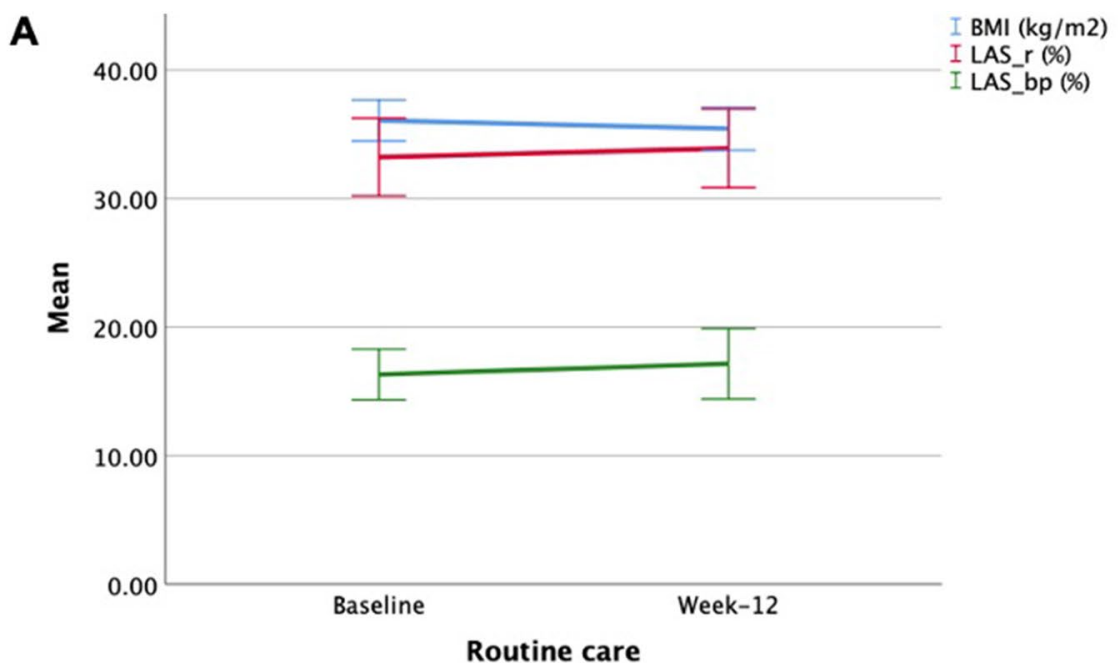

Error Bars: $95 \% \mathrm{Cl}$

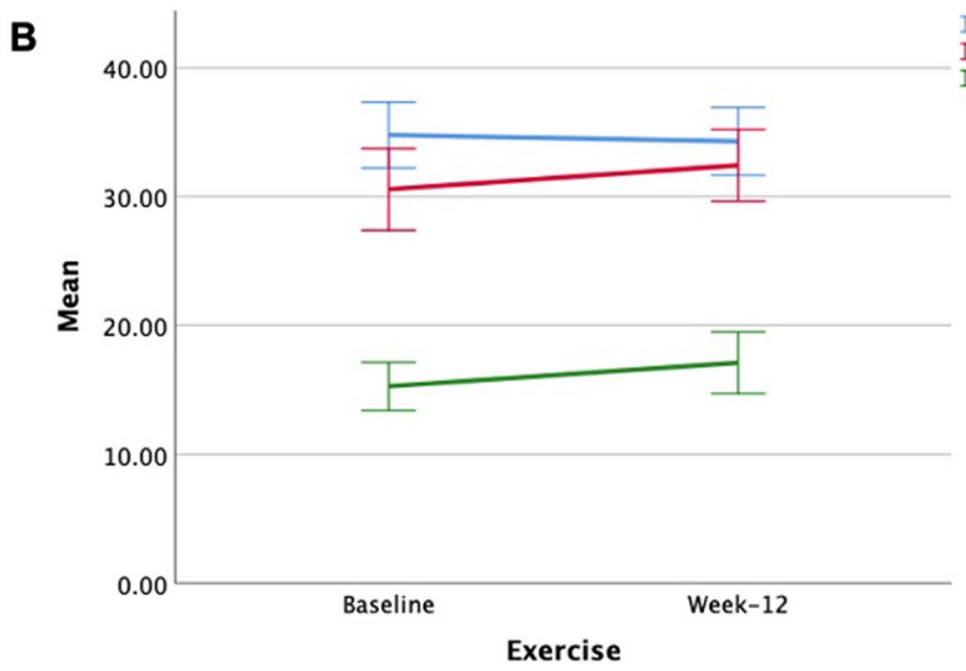

Error Bars: 95\% CI

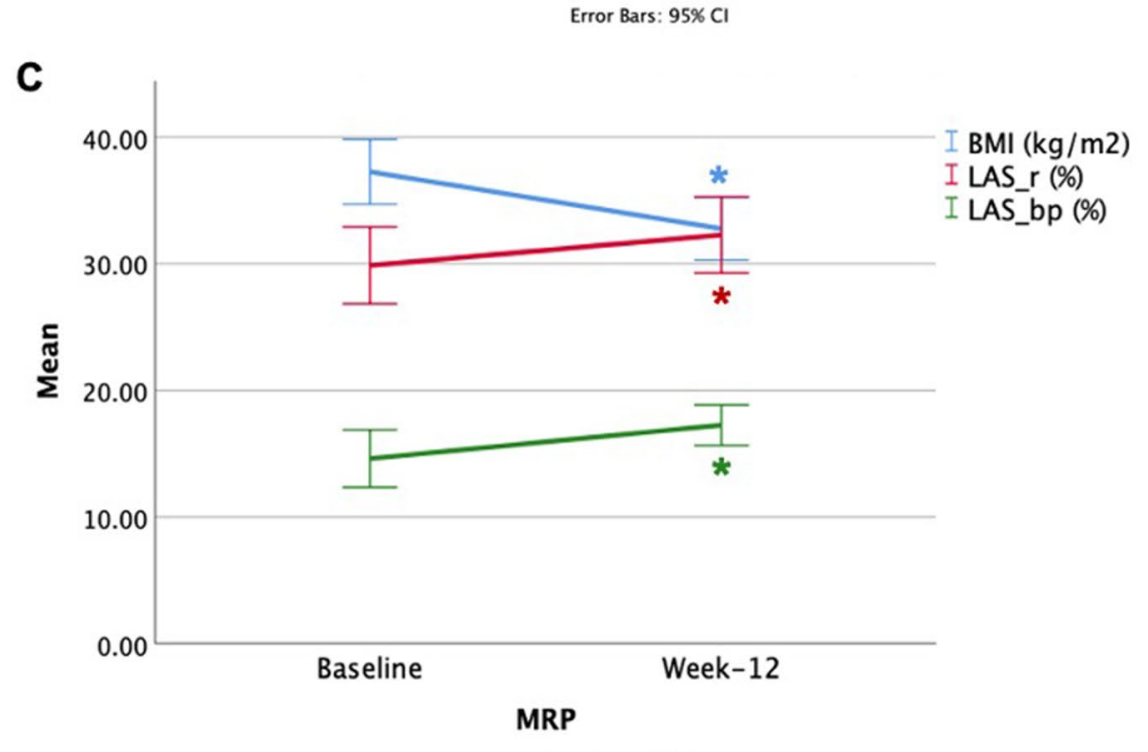

Error Bars: $95 \% \mathrm{Cl}$
BMI $(\mathrm{kg} / \mathrm{m} 2)$

I LAS_r $(\%)$

LAS_bp (\%) 


\section{Changes in LA parameters post-lifestyle intervention}

Our results showed no significant change in LA volumes and EF measured by CMR in any of the trial groups on both between- and within-group analysis. However, LAS showed a within-group increase in the reservoir and boosterpump function in the MRP group only, in combination with a significant reduction in BMI and SBP. As these changes were not statistically significant on between-group analysis, they should be considered hypothesis generating, and could suggest an improvement in LA filling and contractility as a result of the low-calorie diet. The improvement in LA reservoir function following MRP could be explained by increased LA compliance. Studies have shown LV diastolic function improves after weight loss in people with obesity after 6-months of a lifestyle intervention [42, 43]. The current study did not demonstrate an improvement of LV diastolic function in the MRP group, which could be attributed to the short duration. The improvement in the booster pump LAS could be attributed to the Frank-Starling mechanism and the increase of preload as LA filling at reservoir was improved [44-46]. Another possible explanation for this might be the presence of a relationship between LA compliance and excess body weight, possibly linked to myocardial fat accumulation and systemic inflammation. Significant weight loss following the MRP likely contributed to these changes. A recent study compared participants with T2D, only participants with T2D and obesity had significantly lower LAS at reservoir and booster-pump [47].

Together, the present findings confirm that early changes in LA reservoir function assessed by strain may precede changes in conventional volumetric measures in people with T2D and obesity. Weight loss via a low energy MRP could improve LA reservoir function. Although, aerobic exercise showed no significant change in LA function, we previously reported an improvement in LV-PEDSR. Therefore, further studies are needed to investigate the combination of exercise and diet to achieve significant weight loss that may provide optimal reversal of diastolic function, with potential prevention HF in people with T2D and obesity.

\section{Left atrial strain as an imaging biomarker}

The findings of this study suggest that reservoir LAS could be a useful non-invasive marker to detect early LA dysfunction and more interestingly, improvement post-intervention in people with T2D and obesity. Accordingly, if backed by future studies, LAS may have the potential to be used as outcome measure in clinical trials. Moreover, it could be used in combination with LV strain assessment to detect subclinical impairments in cardiac function and provide an opportunity for early intervention to prevent disease progression.

\section{Study limitations}

The DIASTOLIC study limitations have been previously published and included the small sample size, unblinded intervention, short duration of follow-up and the high rate $(19 \%)$ of non-compliance in the exercise group [23]. Although the DIASTOLIC study achieved the trial statistical power, it could be under powered for this post-hoc analysis, which limits the detection of between-groups differences. However, these analyses should be considered hypothesis-generating, and further studies are needed to confirm these novel findings.

\section{Conclusion}

In working-age adults with T2D and obesity, a 12-week lifestyle intervention of low-energy MRP, but not exercise training, led to significant improvements in LA reservoir and booster-pump function assessed by CMR LAS, in conjunction with a significant reduction in BMI and SBP. However, these were not significant on between-group analysis, and should be considered hypothesis-generating.

Supplementary Information The online version contains supplementary material available at https://doi.org/10.1007/s10554-022-02578-z.

Acknowledgements This study was supported by the the National Institute for Health Research (NIHR) Leicester Biomedical Research Centre and NIHR Leicester Clinical Research Facility. The views expressed are those of the authors and not necessarily those of the NHS, the NIHR or the Department of Health and Social Care.

Author contributions We confirm that all authors have participated in the work and have reviewed the final manuscript before submission.

Funding The DIASTOLIC study was funded by NIHR through a career development fellowship (G McCann, CDF 2014-07-045). AA is funded by King Saud bin Abdulaziz University for Health Sciences, Riyadh, Saudi Arabia.

Data availability ClinicalTrials.gov Identifier: NCT02590822.

\section{Declarations}

Conflict of interest The authors report no relationships that could be construed as a conflict of interest.

Ethical approval Ethical approval was granted by the National Research Ethics Service (REC: West Midlands, 15/WM/0222). The study was conducted in accordance with International Conference on Harmonisation-Good Clinical Practice guidelines and the Declaration of Helsinki. All participants provided written informed consent in advance of entering into the study. 
Open Access This article is licensed under a Creative Commons Attribution 4.0 International License, which permits use, sharing, adaptation, distribution and reproduction in any medium or format, as long as you give appropriate credit to the original author(s) and the source, provide a link to the Creative Commons licence, and indicate if changes were made. The images or other third party material in this article are included in the article's Creative Commons licence, unless indicated otherwise in a credit line to the material. If material is not included in the article's Creative Commons licence and your intended use is not permitted by statutory regulation or exceeds the permitted use, you will need to obtain permission directly from the copyright holder. To view a copy of this licence, visit http://creativecommons.org/licenses/by/4.0/.

\section{References}

1. Kannel WB, Hjortland M, Castelli WP (1974) Role of diabetes in congestive heart failure: the Framingham study. Am J Cardiol 34:29-34

2. Maisch B, Alter P, Pankuweit S (2011) Diabetic cardiomyopathy-fact or fiction? Herz 36:102-115

3. Hayat SA, Patel B, Khattar RS, Malik RA (2004) Diabetic cardiomyopathy: mechanisms, diagnosis and treatment. Clin Sci (Lond) 107:539-557

4. Gulsin GS, Athithan L, McCann GP (2019) Diabetic cardiomyopathy: prevalence, determinants and potential treatments. Ther Adv Endocrinol Metab 10:2042018819834869

5. Seferovic PM, Petrie MC, Filippatos GS et al (2018) Type 2 diabetes mellitus and heart failure: a position statement from the Heart Failure Association of the European Society of Cardiology. Eur J Heart Fail 20:853-872

6. Thomas L, Marwick TH, Popescu BA, Donal E, Badano LP (2019) Left atrial structure and function, and left ventricular diastolic dysfunction. J Am Coll Cardiol 73:1961

7. Nagueh SF, Smiseth OA, Appleton CP et al (2016) Recommendations for the evaluation of left ventricular diastolic function by echocardiography: an update from the American Society of Echocardiography and the European Association of Cardiovascular Imaging. J Am Soc Echocardiogr 29:277-314

8. Tsang TS, Barnes ME, Gersh BJ, Bailey KR, Seward JB (2002) Left atrial volume as a morphophysiologic expression of left ventricular diastolic dysfunction and relation to cardiovascular risk burden. Am J Cardiol 90:1284-1289

9. Graça B, Ferreira MJ, Donato P, Gomes L, Castelo-Branco M, Caseiro-Alves F (2014) Left atrial dysfunction in type 2 diabetes mellitus: insights from cardiac MRI. Eur Radiol 24:2669-2676

10. Mondillo S, Cameli M, Caputo ML et al (2011) Early detection of left atrial strain abnormalities by speckle-tracking in hypertensive and diabetic patients with normal left atrial size. J Am Soc Echocardiogr 24:898-908

11. Kadappu KK, Boyd A, Eshoo S et al (2012) Changes in left atrial volume in diabetes mellitus: more than diastolic dysfunction? Eur Heart J Cardiovasc Imaging 13:1016-1023

12. Singh A, Addetia K, Maffessanti F, Mor-Avi V, Lang RM (2017) LA strain for categorization of LV diastolic dysfunction. JACC Cardiovasc Imaging 10:735-743

13. Modin D, Biering-Sørensen SR, Møgelvang R, Alhakak AS, Jensen JS, Biering-Sørensen T (2019) Prognostic value of left atrial strain in predicting cardiovascular morbidity and mortality in the general population. Eur Heart J Cardiovasc Imaging 20:804-815

14. Santos ABS, Roca GQ, Claggett B et al (2016) Prognostic relevance of left atrial dysfunction in heart failure with preserved ejection fraction. Circ Heart Fail 9:e002763
15. Gan GCH, Kadappu KK, Bhat A et al (2021) Left atrial strain is the best predictor of adverse cardiovascular outcomes in patients with chronic kidney disease. J Am Soc Echocardiogr 34:166-175

16. Lin J, Ma H, Gao L et al (2020) Left atrial reservoir strain combined with E/E' as a better single measure to predict elevated LV filling pressures in patients with coronary artery disease. Cardiovasc Ultrasound 18:11

17. Fan JL, Su B, Zhao X et al (2020) Correlation of left atrial strain with left ventricular end-diastolic pressure in patients with normal left ventricular ejection fraction. Int J Cardiovasc Imaging 36:1659-1666

18. Tadic M, Vukomanovic V, Cuspidi C, Suzic-Lazic J, Stanisavljevic D, Celic V (2017) Left atrial phasic function and heart rate variability in asymptomatic diabetic patients. Acta Diabetol 54:301-308

19. Vukomanovic V, Suzic-Lazic J, Celic V et al (2020) Is there association between left atrial function and functional capacity in patients with uncomplicated type 2 diabetes? Int J Cardiovasc Imaging 36:15-22

20. Anderson JW, Kendall CWC, Jenkins DJA (2003) Importance of weight management in type 2 diabetes: review with meta-analysis of clinical studies. J Am Coll Nutr 22:331-339

21. Inzucchi SE, Bergenstal RM, Buse JB et al (2012) Management of hyperglycemia in type 2 diabetes: a patient-centered approach. Diabetes Care 35:1364

22. Fox CS, Golden SH, Anderson C et al (2015) Update on prevention of cardiovascular disease in adults with type 2 diabetes mellitus in light of recent evidence. Circulation 132:691-718

23. Gulsin GS, Swarbrick DJ, Athithan L et al (2020) Effects of lowenergy diet or exercise on cardiovascular function in working-age adults with type 2 diabetes: a prospective, randomized, open-label, blinded end point trial. Diabetes Care 43:1300-1310

24. Gulsin GS, Brady EM, Swarbrick DJ et al (2019) Rationale, design and study protocol of the randomised controlled trial: diabetes interventional assessment of slimming or training to lessen inconspicuous cardiovascular dysfunction (the DIASTOLIC study). BMJ Open 9:e023207

25. Lang RM, Badano LP, Mor-Avi V et al (2015) Recommendations for cardiac chamber quantification by echocardiography in adults: an update from the American Society of Echocardiography and the European Association of Cardiovascular Imaging. J Am Soc Echocardiogr 28:1-39

26. Alfuhied A, Marrow BA, Elfawal S et al (2020) Reproducibility of left atrial function using cardiac magnetic resonance imaging. Eur Radiol 31:1-10

27. Atas H, Kepez A, Atas DB et al (2014) Effects of diabetes mellitus on left atrial volume and functions in normotensive patients without symptomatic cardiovascular disease. J Diabetes Complic $28: 858-862$

28. Jensen Magnus T, Fung K, Aung N et al (2019) Changes in cardiac morphology and function in individuals with diabetes mellitus. Circ Cardiovasc Imaging 12:e009476

29. Skali H, Shah A, Gupta DK et al (2015) Cardiac structure and function across the glycemic spectrum in elderly men and women free of prevalent heart disease: the Atherosclerosis Risk In the Community study. Circ Heart Fail 8:448-454

30. Lindman BR, Dávila-Román VG, Mann DL et al (2014) Cardiovascular phenotype in HFpEF patients with or without diabetes: a RELAX trial ancillary study. J Am Coll Cardiol 64:541-549

31. Gulsin GS, Kanagala P, Chan DCS et al (2019) Differential left ventricular and left atrial remodelling in heart failure with preserved ejection fraction patients with and without diabetes. Ther Adv Endocrinol Metab 10:2042018819861593

32. Kaminski M, Steel K, Jerosch-Herold M et al (2011) Strong cardiovascular prognostic implication of quantitative left atrial contractile function assessed by cardiac magnetic resonance imaging 
in patients with chronic hypertension. J Cardiovasc Magn Reson 13:42

33. Kanagala P, Arnold JR, Cheng ASH et al (2020) Left atrial ejection fraction and outcomes in heart failure with preserved ejection fraction. Int J Cardiovasc Imaging 36:101-110

34. Pellicori P, Zhang J, Lukaschuk E et al (2014) Left atrial function measured by cardiac magnetic resonance imaging in patients with heart failure: clinical associations and prognostic value. Eur Heart J 36:733-742

35. Cameli M, Lisi M, Focardi M et al (2012) Left atrial deformation analysis by speckle tracking echocardiography for prediction of cardiovascular outcomes. Am J Cardiol 110:264-269

36. Habibi M, Chahal H, Opdahl A et al (2014) Association of CMRmeasured LA function with heart failure development: results from the MESA study. JACC Cardiovasc Imaging 7:570-579

37. Huang G, Zhang L, Xie M, Fu M, Huang J, Lv Q (2010) Assessment of left atrial function in diabetes mellitus by left atrial volume tracking method. J Huazhong Univ Sci Technol Med Sci 30:819-823

38. Eshoo S, Ross David L, Thomas L (2009) Impact of mild hypertension on left atrial size and function. Circ Cardiovasc Imaging 2:93-99

39. Tan YT, Wenzelburger F, Lee E et al (2010) Reduced left atrial function on exercise in patients with heart failure and normal ejection fraction. Heart 96:1017

40. Muranaka A, Yuda S, Tsuchihashi K et al (2009) Quantitative assessment of left ventricular and left atrial functions by strain rate imaging in diabetic patients with and without hypertension. Echocardiography 26:262-271
41. Chirinos JA, Sardana M, Satija V et al (2019) Effect of obesity on left atrial strain in persons aged 35-55 years (The Asklepios Study). Am J Cardiol 123:854-861

42. Varli M, Turhan S, Aras S, Atli T, Erdogan G (2010) Effects of weight loss on ventricular systolic and diastolic functions and left ventricular mass assessed by tissue doppler imaging in obese geriatric women: preliminary report. Aging Clin Exp Res 22:206-211

43. Kosmala W, O'Moore-Sullivan T, Plaksej R, Przewlocka-Kosmala M, Marwick TH (2009) Improvement of left ventricular function by lifestyle intervention in obesity: contributions of weight loss and reduced insulin resistance. Diabetologia 52:2306-2316

44. Pagel PS, Kehl F, Gare M, Hettrick DA, Kersten JR, Warltier DC (2003) Mechanical function of the left atrium: new insights based on analysis of pressure-volume relations and doppler echocardiography. Anesthesiology 98:975-994

45. Yamaguchi M, Arakawa M, Tanaka T, Takaya T, Nagano T, Hirakawa S (1987) Study on left atrial contractile performance-participation of Frank-Starling mechanism. Jpn Circ J 51:1001-1009

46. Stefanadis C, Dernellis J, Toutouzas P (2001) A clinical appraisal of left atrial function. Eur Heart J 22:22-36

47. Mohseni-Badalabadi R, Mehrabi-Pari S, Hosseinsabet A (2020) Evaluation of the left atrial function by two-dimensional speckletracking echocardiography in diabetic patients with obesity. Int $\mathrm{J}$ Cardiovasc Imaging 36:643-652

Publisher's Note Springer Nature remains neutral with regard to jurisdictional claims in published maps and institutional affiliations.

\section{Authors and Affiliations}

\section{Aseel Alfuhied ${ }^{1,2,3} \cdot$ Gaurav S. Gulsin $^{1,2} \cdot$ Lavanya Athithan $^{1,2} \cdot$ Emer M. Brady $^{1,2} \cdot$ Kelly Parke $^{1,2} \cdot$ Joseph Henson $^{1,2,4}$. Emma Redman ${ }^{1,2,4}$. Anna-Marie Marsh ${ }^{1,2}$. Thomas Yates ${ }^{1,2,4} \cdot$ Melanie J. Davies ${ }^{4}$. Gerry P. McCann ${ }^{1,2}$. Anvesha Sing ${ }^{1,2}$ (D)}

Aseel Alfuhied

aa1108@1eicester.ac.uk

Gaurav S. Gulsin

gg149@leicester.ac.uk

Lavanya Athithan

la185@leicester.ac.uk

Emer M. Brady

emb29@leicester.ac.uk

Kelly Parke

kelly.parke@uhl-tr.nhs.uk

Joseph Henson

jjh18@le.ac.uk

Emma Redman

eb362@leicester.ac.uk

Anna-Marie Marsh

amm61@ leicester.ac.uk
Thomas Yates

ty20@ leicester.ac.uk

Melanie J. Davies

melanie.davies@uhl-tr.nhs.uk

Gerry P. McCann

gpm12@leicester.ac.uk

1 Department of Cardiovascular Sciences, University of Leicester, Leicester, UK

2 National Institute for Health Research (NIHR) Leicester Biomedical Research Centre, Glenfield Hospital, Groby Road, Leicester LE3 9QP, UK

3 King Saud Bin Abdulaziz University for Health Sciences, Riyadh, Kingdom of Saudi Arabia

4 Diabetes Research Centre, NIHR Leicester Biomedical Research Centre, Leicester, UK 